\title{
Incompletely Stirred Reactor Network Modeling of a Model Gas Turbine Combustor
}

\author{
Savvas Gkantonas* \\ Hopkinson Laboratory, University of Cambridge, Cambridge, CB2 1PZ, UK \\ Andrea Giusti ${ }^{\dagger}$ \\ Faculty of Engineering, Imperial College London, London, SW7 2AZ, UK \\ Epaminondas Mastorakos \\ Hopkinson Laboratory, University of Cambridge, Cambridge, CB2 1PZ, UK
}

\begin{abstract}
The prediction of soot emissions is a requirement for the development of gas turbine combustors. Apart from global quantities related to soot mass, future regulations also call for the control of particle number. Therefore, theoretical models for soot from combustion devices must include various nucleation, growth, and oxidation mechanisms and aerosol physics in order to predict the soot particle number distribution. This paper introduces an approach based on Incompletely Stirred Reactor Network (ISRN) modeling that simplifies calculations and facilitates parametric analyses with very complex soot models. The method is based on the Conditional Moment Closure (CMC) combustion model and Incompletely Stirred Reactor (ISR) theory, which is here extended to a reactor network formulation. An ISR is a volume that is inhomogeneous in terms of mixture fraction but is characterized by homogeneous conditional averages, such as temperature conditioned on the mixture fraction having a particular value. A network of ISRs can then be deployed to separately capture soot production and oxidation regions exhibiting different degrees of micro-mixing rates and residence times, as typically observed in practical combustors. The ISRN approach is demonstrated in this paper for a model aero-engine combustor for which detailed CFD and experimental data exist, and it is found that reasonable accuracy is produced at a significantly reduced computational cost.
\end{abstract}

\section{Nomenclature}

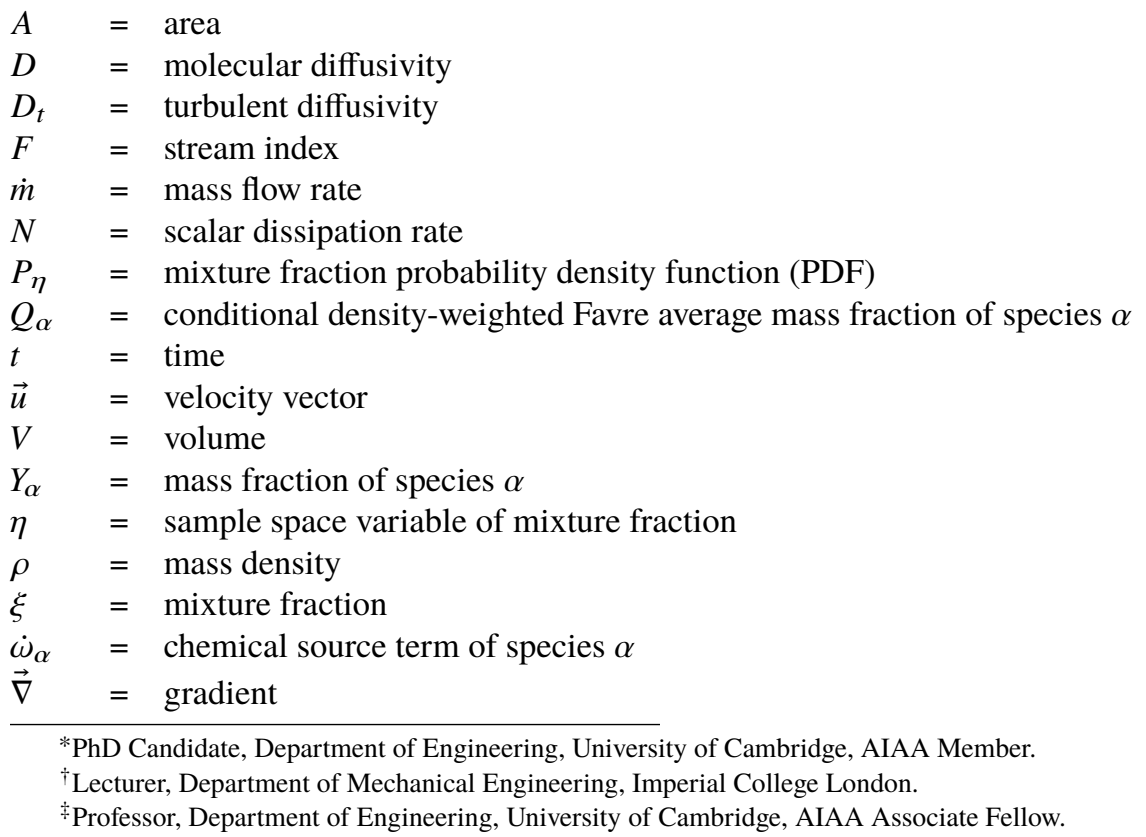




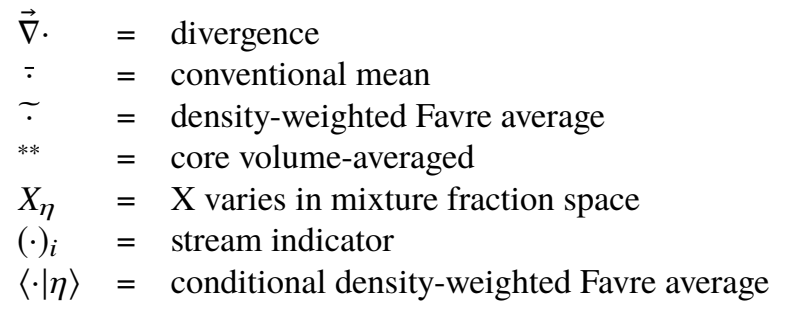

\section{Introduction}

The prediction of soot emissions and control of the particle sizes emitted from aero-engine combustors is of vital importance if next-generation engines are to mitigate the effects of particulate matter to human health and the environment [1]. However, the nature of soot evolution in turbulent flow poses significant numerical challenges. Apart from an accurate knowledge of turbulent mixing and finite-rate chemistry effects, reliable predictions of particulate emissions necessitate the use of complex soot models able to describe the underlying chemistry and the morphology of particles [2]. Predictions are known to be very sensitive to the choice of the soot model [3, 4]; therefore, sensitivity analysis and comparison between soot models are necessary to gain more insights into soot formation and oxidation. Nevertheless, newly appeared comprehensive soot models [5] cannot easily be used in conjunction with high-fidelity CFD simulations to perform parametric studies during the design phase of combustors, given their high dimensionality and computational cost. As a result, there is motivation to develop computationally inexpensive methods that permit the use of complex soot models and the prediction of soot emissions within a reasonable degree of accuracy in both magnitude and trend.

In this direction, this study introduces a new approach utilizing Incompletely Stirred Reactor Network (ISRN) modeling to perform kinetic post-processing of CFD simulations. Due to its simplicity, this methodology allows for the use of comprehensive soot models with modest computational cost, while retaining approximate turbulence-chemistry interaction effects on the sooting flame structure. The Incompletely Stirred Reactor (ISR) model, developed by Bilger and coworkers [9-12], is a generalization of the Perfectly Stirred Reactor. Its derivation is based on the Conditional Moment Closure (CMC) method for turbulent reacting flow [13] and can be viewed as a zero-dimensional (or spatially-integrated) approximation of the full multi-dimensional CMC equation. An ISR is defined as a region of the flow, within which the flow and mixture fraction are inhomogeneous, but conditional averages of reacting scalars, conditioned on the mixture fraction, are uniform. In the limit of strongly recirculating flow, far from extinction, ignition, or other highly-transient phenomena, the latter assumption allows for the use of simple ordinary differential equations in mixture fraction space to model a combustor in its entirety, hence reducing the total computational cost. The approach was initially developed for non-premixed combustion but can be extended to multi-mode combustion following the newest developments of the CMC method [14].

Notwithstanding that the ISR conditions are generally achieved in the primary zone of a highly efficient aeroengine combustor, conditional quantities related to soot evolution show strong spatial dependence, as discussed in Refs. [8, 15, 16]. Therefore, it is necessary to extend the approach to a network of ISRs spanning the whole combustor under investigation. The combustor can then be partitioned in separate soot production and oxidation regions that exhibit different degrees of micro-mixing rates and residence times. In the context of kinetic post-processing, the ISRN equations can be decoupled from the calculation of the flow and mixing fields, consistently with other methods using ideal reactors (e.g. [17]). Unlike ideal reactors, however, unmixedness can be directly taken into account via the scalar dissipation rate and the mixture fraction probability density function. Their quantification is performed through averaging of the mixing field extracted from a reference CFD simulation, which could be of lower detail concerning the combustion and pollutant chemistry hence allowing significant computation time savings.

The main objective of this study is to demonstrate the ability of the ISRN approach to describe soot evolution in a model aero-engine combustor. For this purpose, we apply the approach to the DLR ethylene-based burner at elevated pressure [18, 19], which replicates some of the features of a Rich-Quench-Lean combustor typically used in gas turbines. This paper is structured as follows. The derivation of the ISRN equations is first introduced, followed by the description of the solution strategy used in the computations. This is followed by a brief description of the investigated burner and the reacting field obtained by the reference CFD simulation. Results and key conclusions close the paper. 


\section{Mathematical model}

An ISR is considered to be a volume $V$ within which conditional averages of reacting scalars, conditioned on the mixture fraction, are independent of position and time. In contrast to a perfectly-stirred reactor, which has uniform composition, this allows an ISR to have mixture fraction inhomogeneities. Various versions of the ISR governing equations have appeared in the literature [9-12]. Here we extend the formulation given by Klimenko and Bilger [13] and derive the governing equations for a network of ISR reactors with an arbitrary number of inlet/outlet streams having non-uniform properties. The equations are derived from the CMC equation assuming high Reynolds number and negligible differential diffusion [13]:

$$
\frac{\partial \bar{\rho} Q_{\alpha} \widetilde{P}_{\eta}}{\partial t}+\vec{\nabla} \cdot\left(\bar{\rho}\left\langle\vec{u} Y_{\alpha} \mid \eta\right\rangle \widetilde{P}_{\eta}\right)=\bar{\rho}\left\langle\dot{\omega}_{\alpha} \mid \eta\right\rangle \widetilde{P}_{\eta}-\frac{\partial^{2} \bar{\rho}\langle N \mid \eta\rangle \widetilde{P}_{\eta}}{\partial \eta^{2}} Q_{\alpha}+\bar{\rho}\langle N \mid \eta\rangle \widetilde{P}_{\eta} \frac{\partial^{2} Q_{\alpha}}{\partial \eta^{2}}
$$

where $\eta$ is the sample space variable of the mixture fraction, $\xi, Q_{\alpha} \equiv\left\langle Y_{\alpha} \mid \eta\right\rangle$ is the conditional average mass fraction of a generic species, $\widetilde{P}_{\eta}$ the Favre mean PDF and $N \equiv D \vec{\nabla} \xi \cdot \vec{\nabla} \xi$ the scalar dissipation rate. Here, the angle brackets represent conditional density-weighted Favre averaging.

Considering statistically stationary flow, integration of Eq. (1) over the core volume and application of the flux divergence theorem to the LHS leads to:

$$
\oint_{A} \bar{\rho}\left\langle\vec{u} Y_{\alpha} \mid \eta\right\rangle \widetilde{P}_{\eta} d \vec{A}=\int_{V}\left[\bar{\rho}\left\langle\dot{\omega}_{\alpha} \mid \eta\right\rangle \widetilde{P}_{\eta}-\frac{\partial^{2} \bar{\rho}\langle N \mid \eta\rangle \widetilde{P}_{\eta}}{\partial \eta^{2}} Q_{\alpha}+\bar{\rho}\langle N \mid \eta\rangle \widetilde{P}_{\eta} \frac{\partial^{2} Q_{\alpha}}{\partial \eta^{2}}\right] d V
$$

By definition, conditional reactive scalar statistics are considered uniform inside an ISR core. Hence, they can be moved out of the integral on the RHS of Eq. (2). Moreover, conditional statistics are only a function of mixture fraction. Therefore, their partial derivatives may be transformed into ordinary ones. Next, by introducing a diffusion approximation for the conditional correlations between species and velocity [13], assuming a turbulent diffusivity $D_{t}$ equal for all scalars, the governing equation can be written in the following discrete form:

$$
\sum_{i=1}^{F^{\text {out }}}\left(\dot{m} \widetilde{P}_{\eta} Q_{\alpha}\right)_{i}-\sum_{j=1}^{F^{i n}}\left(\dot{m} \widetilde{P}_{\eta} Q_{\alpha}\right)_{j}+\sum_{i=1}^{F}\left(\bar{\rho} D_{t}\left(\vec{\nabla} Q_{\alpha}\right) \widetilde{P}_{\eta} \vec{A}\right)_{i}=V \rho^{* *}\left[P_{\eta}^{* *}\left(\dot{\omega}_{\alpha} \mid \eta+N_{\eta}^{* *} \frac{d^{2} Q_{\alpha}}{d \eta^{2}}\right)-Q_{\alpha} \frac{d^{2} N_{\eta}^{* *} P_{\eta}^{* *}}{d \eta^{2}}\right]
$$

where the core volume-averaged mass density, $\rho^{* *}$, mixture fraction PDF, $P_{\eta}^{* *}$, and scalar dissipation rate, $N_{\eta}^{* *}$ are defined by:

$$
\begin{gathered}
\rho^{* *} \equiv \frac{1}{V} \int_{V} \bar{\rho} d V \\
P_{\eta}^{* *} \equiv \frac{1}{V \rho^{* *}} \int_{V} \bar{\rho} \widetilde{P}_{\eta} d V \\
N_{\eta}^{* *} \equiv \frac{1}{V \rho^{* *} P_{\eta}^{* *}} \int_{V} \bar{\rho}\langle N \mid \eta\rangle P_{\eta} d V
\end{gathered}
$$

Note the last term on the RHS of Eq. (3). To close this term, the transport equation for the mixture fraction PDF may be utilized [13]. Given a statistically stationary flow and neglecting molecular fluxes, the PDF equation becomes:

$$
\vec{\nabla} \cdot\left(\bar{\rho}\langle\vec{u} \mid \eta\rangle \widetilde{P}_{\eta}\right)=-\frac{\partial^{2} \bar{\rho}\langle N \mid \eta\rangle \widetilde{P}_{\eta}}{\partial \eta^{2}} \Rightarrow \sum_{i=1}^{F^{\text {out }}}\left(\dot{m} \widetilde{P}_{\eta}\right)_{i}-\sum_{j=1}^{F^{i n}}\left(\dot{m} \widetilde{P}_{\eta}\right)_{j}=-V \rho^{* *} \frac{d^{2} N_{\eta}^{* *} P_{\eta}^{* *}}{d \eta^{2}}
$$

where the flux divergence theorem and the definitions of Eq. (446) are employed. Consistently with the stirred reactor concept, the conditional quantities exiting the ISR may be taken equal to conditional quantities in the core. However, it is not necessary to assume that the outlet streams PDF are identical to the core [12]. Finally, the combination of Eq. (3)-17) leads to the governing equation of an ISRN element:

$$
\frac{1}{V \rho^{* *} P_{\eta}^{* *}}\left[\sum_{j=1}^{F^{i n}}\left(\dot{m} \widetilde{P}_{\eta}\right)_{j}\left[Q_{\alpha}-\left(Q_{\alpha}\right)_{j}\right]+\sum_{i=1}^{F}\left(\bar{\rho} D_{t}\left(\vec{\nabla} Q_{\alpha}\right) \widetilde{P}_{\eta} \vec{A}\right)_{i}\right]=\left\langle\dot{\omega}_{\alpha} \mid \eta\right\rangle+N_{\eta}^{* *} \frac{d^{2} Q_{\alpha}}{d \eta^{2}}
$$

Here, it important to note that Eq. 8 8 is identical to the governing equation of Ref. [13] when the second term on the LHS is removed, and a single ISR reactor is employed having a single inlet and outlet stream. 


\section{Solution strategy}

The ISRN approach starts with the creation of a standard computational grid and the calculation of the average reacting flow field using CFD. The modeling approach for the reference CFD simulation can vary given the availability of resources, but care must be taken for each specific application, so the underlying mixing field is well captured. Existing methods may include, for example, the use of RANS or LES coupled with the flamelet approach, CMC, transported PDF, or the Eddy Dissipation concept [20].

The ISRN equations are then solved using an in-house unstructured finite volume code, initially developed for CMC modeling (see [7, 16, 21] and references therein). Following the practice in the pre-existing code, the ISRN is discretized on a coarse mesh, which is reconstructed around the grid of the reference CFD simulation. The latter allows for arbitrary positioning of the ISR reactors and facilitates data transfer between solvers by exploiting the topology of the CFD faces. In contrast with ideal reactor network approaches (e.g. [17]), clustering procedures are not required to identify chemically and physically homogeneous zones since ISRs are inhomogeneous in terms of their flow and mixture fraction fields. The current implementation also permits the use of parallel evaluation of reactors, hence speeding up computations.

The evaluation of core volume-averaged quantities and area-averaged fluxes requires knowledge about the mean flow and mixing fields. In this work, these quantities are extracted from a LES-CMC simulation, which will be described later. CFD derived quantities are either evaluated at the faces of ISR reactors to calculate LHS terms of Eq. (8) or used during core volume-averaging. The procedure is very similar to the one followed every time-step in existing CMC codes (e.g. [21]). After the end of the calculation, unconditional quantities may be derived through the integration of the mixture fraction PDF. Here, unconditional values are calculated at the CFD grid after applying an inverse square distance interpolation over neighbor ISR reactors. The PDF is here modeled with a presumed $\beta$-function computed from the mixture fraction, $\widetilde{\xi}$, and the mixture fraction variance, $\widetilde{\xi^{\prime \prime 2}}$ of each CFD cell. In the case of singularities at the $\eta$-space boundaries, the $\beta$-function is replaced by a delta function.

The core-volume averaged scalar dissipation rate is obtained through Eq. (6). To calculate the conditional scalar dissipation rate at the CFD level, the Amplitude Mapping Closure (AMC) model [22] is employed, i.e. $\langle N \mid \eta\rangle=N_{0} G_{\eta}$ where $G_{\eta}$ and $N_{0}$ are $G_{\eta}=\exp \left(-2\left[\operatorname{erf}^{-1}(2 \eta-1)\right]^{2}\right)$ and $N_{0}=\widetilde{N} / \int_{0}^{1} \widetilde{P_{\eta}} G_{\eta} d \eta$. The average scalar dissipation rate, $\widetilde{N}$, is readily available from the CFD simulation. It is important to note that in contrast with existing ISR models [9-12], here, the core-volume averaged scalar dissipation rate is not based on the double integration of Eq. (7), raising issues of consistency with the PDF transport equation. Nonetheless, it has been observed that in a multi-dimensional system such as an ISRN, this approach may result in negative values of the scalar dissipation rate being obtained through machine precision errors when the PDF tends to zero [13]. Here, we preferred a more robust approach in evaluating the conditional scalar dissipation rate, which is also consistent with state-of-the-art CMC approaches (e.g. [23]).

An operator splitting technique is implemented for the solution of the ISRN equations. Transport in physical space, i.e. LHS terms of Eq. (8), are solved first, followed by diffusion in mixture fraction space and the chemical source term integration. The chemical source term is closed with a first-order approximation. A short description of the chemical mechanism and the soot model used in this work is given in the following. A first-order upwind scheme is used for the evaluation of conditional gradients, whereas the diffusion term in mixture fraction space is discretized with a second-order scheme. The inert mixing solution is imposed at the inlets, and reactors are initialized with a fully-burning solution. Both walls and outlets are modeled with a zero-gradient condition. Consistently with the reference CFD simulation, mixture fraction space is discretized using 51 bins clustered around the stoichiometric mixture fraction ( $\eta_{s t}=0.063$ for ethylene). Pure air is imposed at $\eta=0$, whereas $\eta=1$ corresponds to pure fuel. Ambient temperature, equal to $298 \mathrm{~K}$, is used for both boundaries, and a constant pressure condition of 3 bar is used for all reactors.

A detailed mechanism with 57 species and 364 reactions for ethylene combustion is employed. The mechanism has been developed by H. Curran at NUI Galway and is derived from the Aramco 1.3 mechanism [24]. The gaseous phase chemistry is then coupled with a simple two-equation semi-empirical model by Leung et al. [25], which has been broadly used in the literature and is based on the solution of the soot mass fraction, $Y_{S}$, and soot number density, $N_{s}$. The soot chemistry reaction rates used in this study can be found in Ref. [26]. For this study, we do not attempt the use of more complex chemical mechanisms or advanced soot models. The same mechanism and soot models are employed for both the ISRN and the reference simulation. Here, the focus is on directly comparing the simulation results of the ISRN and the reference CFD simulation to assess the ability of the ISRN in replicating mixing and finite-rate chemistry effects on soot evolution. 
Table 1 Operating conditions of the investigated case [18]

\begin{tabular}{ccccccc}
\hline $\begin{array}{c}\text { Pressure } \\
\text { (bar) }\end{array}$ & $\phi$ & $\phi_{\text {global }}$ & $\begin{array}{c}\dot{V}_{\text {fuel }} \\
(\mathrm{slpm})\end{array}$ & $\begin{array}{c}\dot{V}_{\text {central,air }} \\
(\text { slpm })\end{array}$ & $\begin{array}{c}\dot{V}_{\text {ring,air }} \\
(\text { slpm })\end{array}$ & $\begin{array}{c}\dot{V}_{\text {secondary,air }} \\
(\text { slpm })\end{array}$ \\
\hline 3 & 1.2 & 0.86 & 39.3 & 140.8 & 328.5 & 187.4 \\
\hline
\end{tabular}

\section{Investigated burner and CFD analysis}

The experimental configuration used in this study is based on a model aero-engine combustor configuration, developed at DLR [18]. The burner can operate at elevated pressures and runs on ethylene fuel. The main combustion chamber measures $120 \mathrm{~mm}$ in height and has a cross-sectional area of $68 \times 68 \mathrm{~mm}^{2}$. Fuel is introduced through 60 annular straight-channel inlets, located in between two concentric nozzles entraining oxidation air with tangential velocity generated by a pair of swirlers. A set of additional injector ports is situated at the height of $80 \mathrm{~mm}$ that radially introduces secondary air into the combustion chamber, consistently with the RQL concept. The computational domain reproducing the experimental rig is schematically shown in Fig. 1.

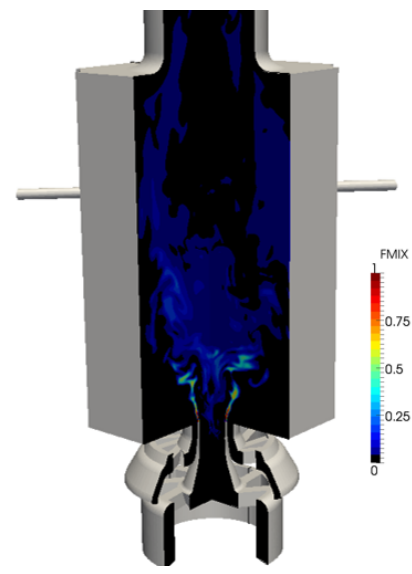

(a) Computational domain with instantaneous mixture fraction field

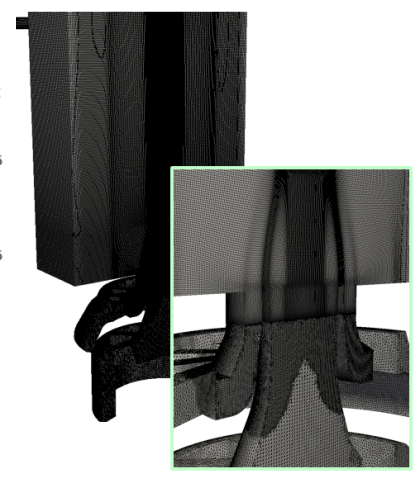

(b) LES mesh

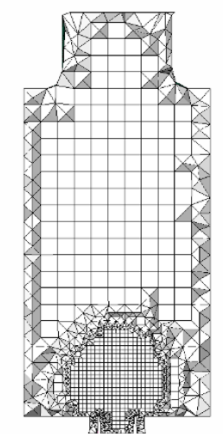

(c) CMC mesh

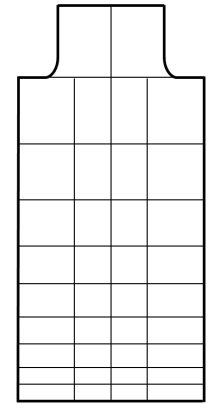

(d) ISRN

Fig. 1 Computational setup details of the LES-CMC and the ISRN simulations.

In this study, a single case, characterized by the operating conditions of Table 1 , is investigated. This case is one of the preferred target flames of the International Sooting Flames (ISF) workshop and has been characterized by various experimental techniques, such as Laser-Induced Incandescence (LII), Particle Image Velocimetry (PIV) and Coherent Anti-Stokes Raman Scattering (CARS) measurements [18, 19, 27].

Together with ISRN calculations for this combustor, a reference CFD simulation using LES and the CMC combustion model has been performed. This simulation was necessary to provide the ISRN with the mean velocity and mixing fields but also to assess the predictability of the ISRN. The LES-CMC approach is based on the time-resolved solution of the local flame structure in mixture fraction space and incorporates molecular mixing and turbulent transport effects. This allows for the reproduction of multi-scale turbulence-chemistry-soot interactions and to properly account for history and finite-rate chemistry effects on soot evolution. For this simulation, the LES equations were solved in a hybrid mesh (fully hexahedral in the combustion chamber and tetrahedral in the air swirler system) of about 30 million cells with a maximum grid size of about $0.2 \mathrm{~mm}$ in the flame region and local refinements near the injection ports. The complete system, including the air swirlers, was included in the simulation. Uniform velocity profiles were imposed at the inlets. A constant pressure condition of 3 bar was used at the outlet, and all solid surfaces were modeled as constant temperature walls with the no-slip condition. CMC equations were solved in a polyhedral mesh of about 65,000 cells with refinement in the flame region. The LES and CMC grids used in the simulation are shown in Fig. 1. The same solution techniques presented in Ref. [16] were employed. Statistics were collected over $80 \mathrm{~ms}$.

In the following, data extracted from LES-CMC are compared against the experimental measurements of Ref. [18, 19, 27]. Figure 2 shows the comparison between axial, radial, and tangential velocity profiles at various heights of the 
burner for a stream-wise cross-section of the flow. In general, numerical results generally show a very good agreement with experimental data and capture all the major features of the measured flow field, which is important for predicting the flame location. The simulation captures the location of the stagnation points and the extent of the recirculation zone across the whole combustion chamber. A very good agreement is also observed in the tangential components indicating that the injection velocity angle from the swirl nozzles is well captured. The time-averaged mean temperature profile, as predicted by LES-CMC, is compared to the experiment in Fig. 3. The simulation predicts a higher drop in temperature near the injection ports which is probably caused by an over-prediction of the back-flow in that region. Moreover, the simulation shows a stronger drop in temperature at the location of the secondary air jets compared to the experiment. Nonetheless, also taking into account the uncertainties of the measurements, the agreement of the predicted to the experimental temperature profile is fairly accurate.

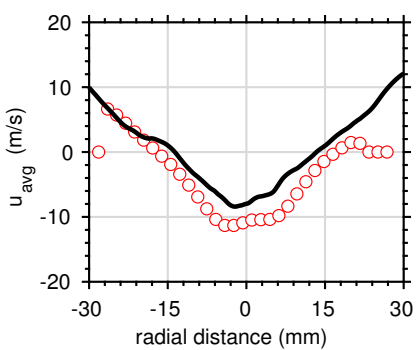

(a) Axial velocity at $70 \mathrm{~mm}$

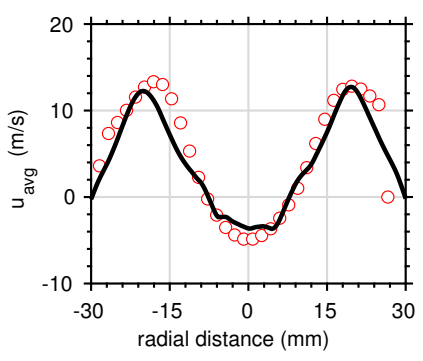

(d) Axial velocity at $20 \mathrm{~mm}$

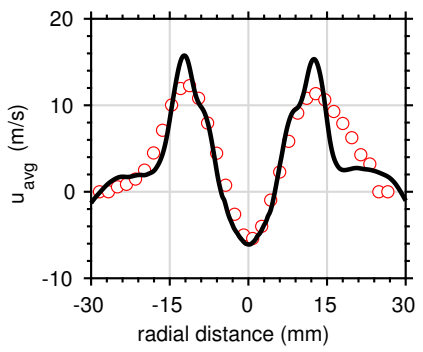

(g) Axial velocity at $9.77 \mathrm{~mm}$

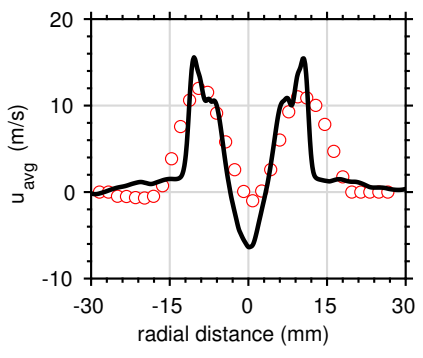

(j) Axial velocity at $4.62 \mathrm{~mm}$

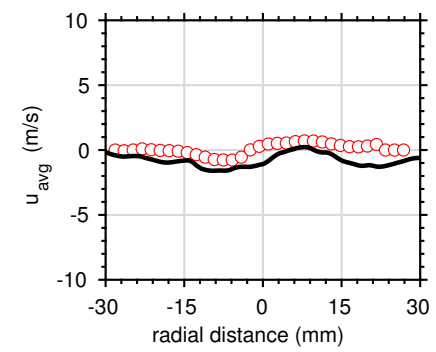

(b) Radial velocity at $70 \mathrm{~mm}$

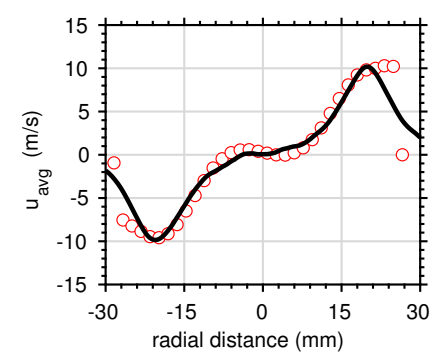

(e) Radial velocity at $20 \mathrm{~mm}$

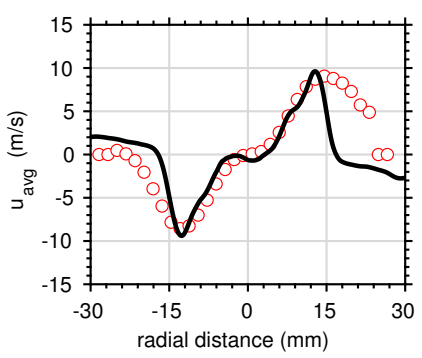

(h) Radial velocity at $9.77 \mathrm{~mm}$

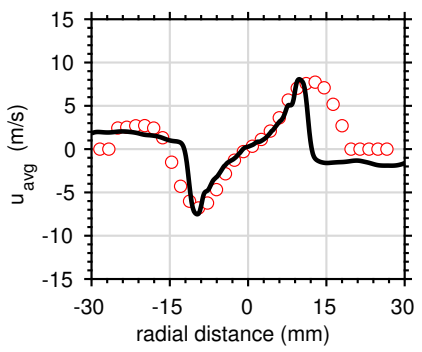

(k) Radial velocity at $4.62 \mathrm{~mm}$

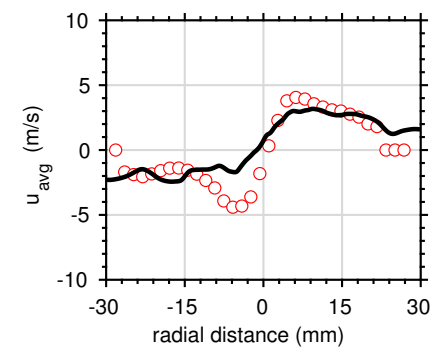

(c) Tangential velocity at $70 \mathrm{~mm}$

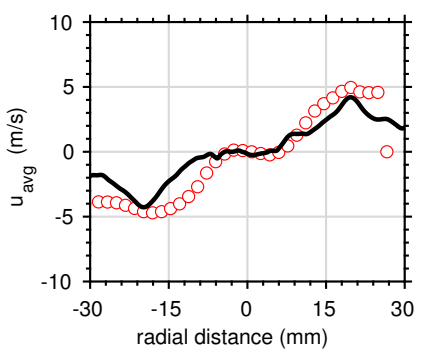

(f) Tangential velocity at $20 \mathrm{~mm}$

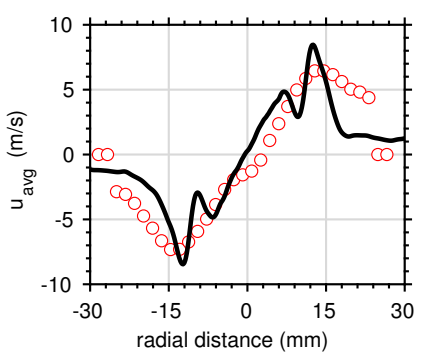

(i) Tangential velocity at $9.77 \mathrm{~mm}$

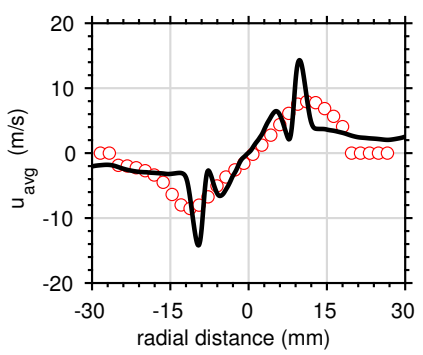

(l) Tangential velocity at $4.62 \mathrm{~mm}$

Fig. 2 Comparison between time-averaged predicted (lines) and experimentally measured (points) velocity profiles at various heights of the combustion chamber. 


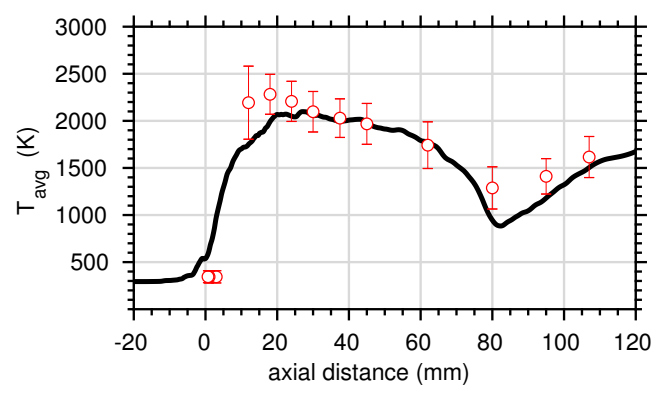

Fig. 3 Comparison between time-averaged predicted (lines) and experimentally measured (points) axial temperature profile.

Once the average mixing and flow fields from the LES-CMC simulation are available, the ISRN approach is then applied to the same flame. For this study, the burner is partitioned in 152 ISR reactors uniformly positioned in the crosswise directions. A cut of the ISRN grid is shown in Fig. 1. It is important to note that ISRN predictions are sensitive to the choice of reactor spacing, but a parametric analysis was out of the scope of this study. Instead, the main objective is to provide a rough and quick estimate of the soot emission in this combustor. The number of reactors employed here was found to be a good compromise between accuracy and speed, as will be demonstrated later. A more refined spacing, aiming to better resolve the gradients of conditional quantities in the soot production and oxidation regions, is expected to improve soot predictions generally.

\section{Results and discussion}

Figure 4 shows the comparison between the experimentally measured and the predicted soot volume fraction from the LES-CMC and ISRN. From the experiment, the majority of soot appears to be generated in the shear layers and located in regions close to the walls where the outer- and inner-recirculation zones meet, raising the total residence time and enhancing soot production [19]. Both simulations accurately capture this effect. Soot is also present further downstream, closely attached to the walls where strain rate and temperature conditions appear to be favorable. Soot is absent for the most part of the inner recirculation zone, possibly due to the enhanced soot oxidation caused by the injection of secondary air. Soot is quickly oxidized before being entrained inside the inner-recirculation zone (IRZ), where residence time would otherwise be suitable for soot production. This is replicated by the simulations. At a region upstream, however, the experiments show a peak in the IRZ, which is not accurately captured by LES-CMC. This is probably related to an over-prediction of the oxidation rate as estimated by the soot model employed here or a soot entrainment time-scale, which is not captured in the simulation time. In addition, soot evolution is very sensitive to the mixture fraction field, for which there is no experimental data. A slight change in the predicted mixture fraction, e.g. as a result of modified boundary conditions, might affect the prediction in this region. The ISRN shows a higher peak in the same region, but the local soot volume fraction scales with the peak soot volume fraction in the combustion chamber, which is higher for the ISRN.

Nonetheless, both LES-CMC and ISRN methods show very promising potential in capturing soot location even with a simplified soot model. These methods are comparable with approaches of other researchers using different turbulent combustion closure methods with more advanced soot models [4, 28-31]. In terms of magnitude, however, both simulations over-predict the total soot volume fraction, as also observed with other studies using semi-empirical two-equation soot models [32, 33] in this burner. The latter is closely related to the choice of the soot model, which is known to affect soot predictions significantly (e.g. [4]). Especially the selection of an acetylene-based two-equation model, like the one used here, generally leads to higher values of soot volume fraction [34] and significantly different predictions than with models using aromatics precursor chemistry [4].

In particular, the ISRN predictions generally show a higher soot volume fraction than LES-CMC, stemming from the off-line approach used in the ISRN. Provided the mean flow and mixing fields, the evolution of conditional scalars, including soot volume fraction and temperature, is inherently different from LES-CMC since no two-way coupling with the reacting flow is attained and excursions of the scalar dissipation rate are not considered. However, the ISRN contains effects of micro-mixing and transport that are especially important during soot evolution; hence, an adequate balance between reaction-transport-diffusion is established. The latter is especially useful for parametric analysis and the search for soot tendency trends. 

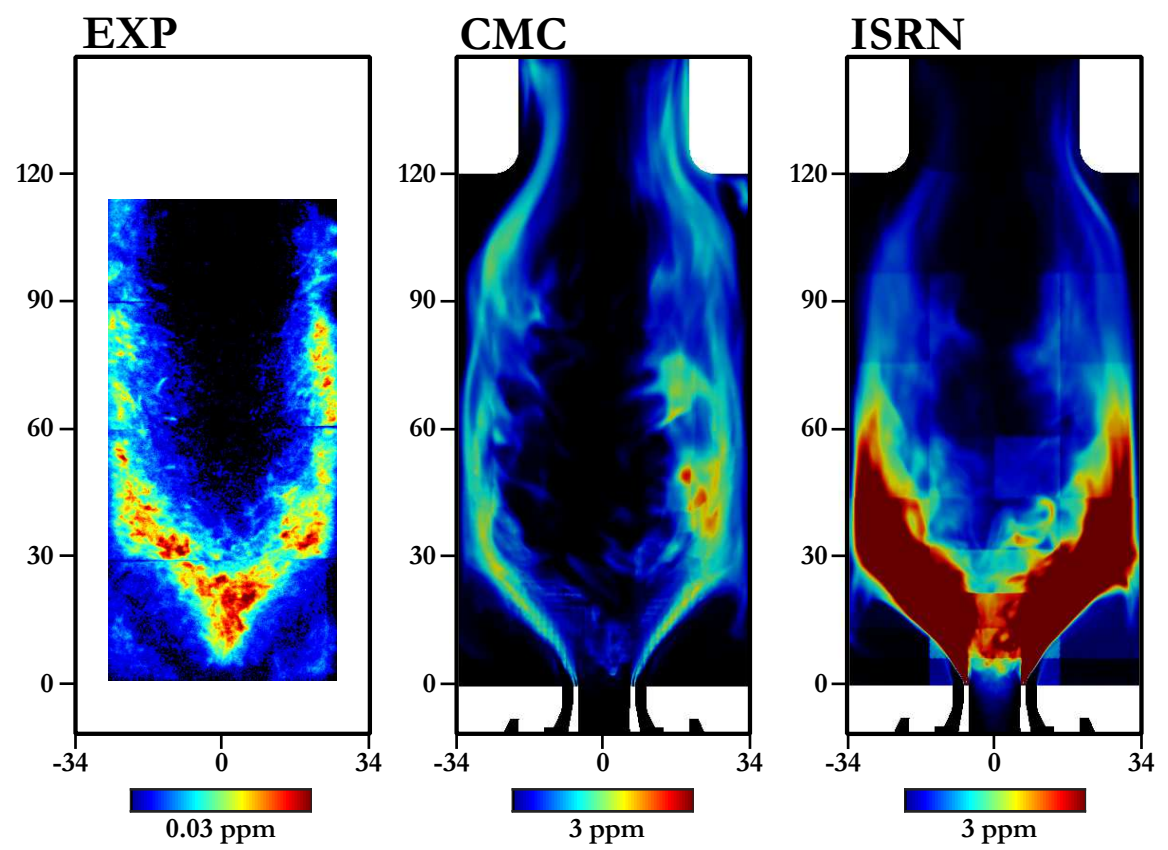

Fig. 4 Comparison between time-averaged experimental and simulated soot volume fraction distributions. Dimensions are in $\mathbf{m m}$.

As far as the implementation aspects of the ISRN are concerned, the method is computationally very efficient. As the mixing field is pre-calculated and only a subset of processes is solved at each time-step, the computational cost is drastically minimized, allowing for the use of even more complex chemical mechanisms and soot models. For the numerical setup presented here, an average runtime for advancing the ISRN model by one time-step $\left(\delta t=2 \times 10^{-6} \mathrm{~s}\right)$ on 1 node (32 MPI processes) of an Intel Xeon Skylake supercomputer is approximately $1.5 \mathrm{~s}$ with $95 \%$ of the time spent for the chemistry integration, similar to laminar flamelet computations. This corresponds to less than $24 \mathrm{~h}$ of physical time for simulation of $100 \mathrm{~ms}$, but the total runtime also scales inversely with the number of total MPI processes. The same performance can also be achieved in a multi-core workstation but is bounded by the available computer memory necessary to process the underlying CFD computation and the total number of scalars.

\section{Conclusions}

A novel approach utilizing Incompletely Stirred Reactor Network (ISRN) modeling has been introduced and formulated. An ISRN comprises a network of stirred reactors that may be positioned and clustered around the mesh of a reference CFD simulation providing the average mixing field. Due to its simplicity and low computational cost, this approach may be used to explore soot evolution in aero-engine combustors employing complex chemical mechanisms and comprehensive soot models that otherwise would be intractable to couple with detailed CFD methods. The ISRN approach is here demonstrated on a calculation of a well-characterized model aero-engine combustor. Results from a reference LES-CMC simulation coupled with detailed chemistry and a semi-empirical two-equation soot model are first presented. A very good agreement with experiments in terms of velocity, temperature, and soot location is observed. The LES-CMC results are then compared with ISRN predictions using the same underlying chemistry and soot model. The comparison provides a satisfactory assessment of the method reliability, demonstrating the ability of the ISRN method to replicate soot emission both in terms of mean location and magnitude while ensuring high computational efficiency. Future work will focus on the comparison with comprehensive soot models and the prediction of particle size distribution.

\section{Acknowledgments}

This work was supported by the UK Engineering and Physical Sciences Research Council (EPSRC) and Rolls-Royce Group. Prof. H. Curran is acknowledged for providing the chemical mechanism for ethylene. The authors are grateful 
to Dr M. Staufer and Dr R. Eggels from Rolls-Royce Deutschland for providing the CFD mesh. The authors would also like to thank Dr K.P. Geigle for the useful discussions regarding the experiment and for providing the experimental data.

\section{References}

[1] D’Anna, A., "Combustion-formed nanoparticles," Proceedings of the Combustion Institute, Vol. 32, No. 1, 2009 , pp. $593-613$. https://doi.org/10.1016/j.proci.2008.09.005

[2] Wang, H., "Formation of nascent soot and other condensed-phase materials in flames," Proceedings of the Combustion Institute, Vol. 33, No. 1, 2011, pp. 41-67. https://doi.org/10.1016/j.proci.2010.09.009.

[3] Roy, S. P., and Haworth, D. C., "A Systematic Comparison of Detailed Soot Models and Gas-Phase Chemical Mechanisms in Laminar Premixed Flames," Combustion Science and Technology, Vol. 188, No. 7, 2016, pp. 1021-1053. https://doi.org/10. 1080/00102202.2016.1145117

[4] Chong, S. T., Raman, V., Mueller, M. E., Selvaraj, P., and Im, H. G., "Effect of soot model, moment method, and chemical kinetics on soot formation in a model aircraft combustor," Proceedings of the Combustion Institute, Vol. 37, No. 1, 2019, pp. 1065 - 1074. https://doi.org/10.1016/j.proci.2018.06.093

[5] Sewerin, F., and Rigopoulos, S., "An LES-PBE-PDF approach for predicting the soot particle size distribution in turbulent flames," Combustion and Flame, Vol. 189, 2018, pp. 62-76. https://doi.org/10.1016/j.combustflame.2017.09.045

[6] Rodrigues, P., Franzelli, B., Vicquelin, R., Gicquel, O., and Darabiha, N., "Coupling an LES approach and a soot sectional model for the study of sooting turbulent non-premixed flames," Combustion and Flame, Vol. 190, 2018, pp. 477-499. https://doi.org/10.1016/j.combustflame.2017.12.009

[7] Gkantonas, S., Sirignano, M., Giusti, A., D’Anna, A., and Mastorakos, E., "Comprehensive Soot Particle Size Distribution Modelling Of A Model Rich-Quench-Lean Burner," 11th Mediterannean Combustion Symposium, Tenerife, Spain, 2019. https://doi.org/10.17863/CAM.43221.

[8] Gkantonas, S., Sirignano, M., Giusti, A., D’Anna, A., and Mastorakos, E., “Comprehensive Soot Particle Size Distribution Modelling Of A Model Rich-Quench-Lean Burner,” Submitted to Fuel, 2019.

[9] Smith, N., "Development of the conditional moment closure method for modelling turbulent combustion," Ph.D. thesis, University of Sydney, 1994.

[10] Mobini, K., "An investigation of the imperfectly stirred reactor modelling of recirculating combustion flows," Ph.D. thesis, University of Sydney, 1998.

[11] Mobini, K., and Bilger, R. W., "Imperfectly Stirred Reactor Model Predictions Of Reaction In A Burner With Strong Recirculation," Combustion Science and Technology, Vol. 176, No. 1, 2004, pp. 45-70. https://doi.org/10.1080/00102200490255334

[12] Mobini, K., and Bilger, R., "Parametric study of the Incompletely Stirred Reactor modeling," Combustion and Flame, Vol. 156, No. 9, 2009, pp. 1818-1827. https://doi.org/10.1016/j.combustflame.2009.06.017

[13] Klimenko, A. Y., and Bilger, R. W., "Conditional moment closure for turbulent combustion,” Progress in energy and combustion science, Vol. 25, No. 6, 1999, pp. 595-687.

[14] Sitte, M. P., and Mastorakos, E., "Large Eddy Simulation of a spray jet flame using Doubly Conditional Moment Closure," Combustion and Flame, Vol. 199, 2019, pp. 309 - 323. https://doi.org/10.1016/j.combustflame.2018.08.026

[15] Attili, A., Bisetti, F., Mueller, M. E., and Pitsch, H., "Formation, growth, and transport of soot in a three-dimensional turbulent non-premixed jet flame," Combustion and Flame, Vol. 161, No. 7, 2014, pp. 1849-1865. https://doi.org/10.1016/j.combustflame. 2014.01 .008

[16] Giusti, A., Gkantonas, S., Foale, J. M., and Mastorakos, E., "Numerical Investigation of Flame Structure and Soot Formation in a Lab-Scale Rich-Quench-Lean Burner," Proceedings of the ASME Turbo Expo 2018: Turbomachinery Technical Conference and Exposition. Volume 4B: Combustion, Fuels, and Emissions, ASME, Oslo, Norway, 2018, p. V04BT04A032. https://doi.org/10.1115/GT2018-76705

[17] Lyra, S., and Cant, R., "Analysis of high pressure premixed flames using Equivalent Reactor Networks for predicting NOx emissions,” Fuel, Vol. 107, 2013, pp. 261 - 268. https://doi.org/10.1016/j.fuel.2012.12.066 
[18] Peter Geigle, K., Hadef, R., and Meier, W., "Soot Formation and Flame Characterization of an Aero-Engine Model Combustor Burning Ethylene at Elevated Pressure," Journal of Engineering for Gas Turbines and Power, Vol. 136, No. 2, 2013, pp. 021505-021505-7. https://doi.org/10.1115/1.4025374

[19] Geigle, K. P., Hadef, R., Stöhr, M., and Meier, W., "Flow field characterization of pressurized sooting swirl flames and relation to soot distributions," Proceedings of the Combustion Institute, Vol. 36, No. 3, 2017, pp. 3917-3924. https://doi.org/10.1016/j.proci.2016.09.024

[20] Giusti, A., and Mastorakos, E., “Turbulent Combustion Modelling and Experiments: Recent Trends and Developments," Flow, Turbulence and Combustion, 2019. https://doi.org/10.1007/s10494-019-00072-6

[21] Zhang, H., Garmory, A., Cavaliere, D. E., and Mastorakos, E., "Large Eddy Simulation/Conditional Moment Closure modeling of swirl-stabilized non-premixed flames with local extinction," Proceedings of the Combustion Institute, Vol. 35, No. 2, 2015, pp. 1167 - 1174. https://doi.org/10.1016/j.proci.2014.05.052

[22] O'Brien, E. E., and Jiang, T., “The conditional dissipation rate of an initially binary scalar in homogeneous turbulence,” Physics of Fluids A: Fluid Dynamics, Vol. 3, No. 12, 1991, pp. 3121-3123. https://doi.org/10.1063/1.858127

[23] Giusti, A., and Mastorakos, E., "Detailed chemistry LES/CMC simulation of a swirling ethanol spray flame approaching blow-off," Proceedings of the Combustion Institute, Vol. 36, No. 2, 2017, pp. 2625-2632. https://doi.org/10.1016/j.proci.2016.06.035

[24] Metcalfe, W. K., Burke, S. M., Ahmed, S. S., and Curran, H. J., "A Hierarchical and Comparative Kinetic Modeling Study of C1,C2 Hydrocarbon and Oxygenated Fuels," International Journal of Chemical Kinetics, Vol. 45, No. 10, 2013, pp. 638-675. https://doi.org/10.1002/kin.20802.

[25] Leung, K. M., Lindstedt, R. P., and Jones, W. P., "A simplified reaction mechanism for soot formation in nonpremixed flames," Combustion and Flame, Vol. 87, No. 3, 1991, pp. 289 - 305. https://doi.org/10.1016/0010-2180(91)90114-Q

[26] Kronenburg, A., Bilger, R. W., and Kent, J. H., "Modeling soot formation in turbulent methane-air jet diffusion flames," Combustion and Flame, Vol. 121, No. 1, 2000, pp. 24 - 40. https://doi.org/10.1016/S0010-2180(99)00146-7.

[27] Geigle, K. P., Köhler, M., O’Loughlin, W., and Meier, W., "Investigation of soot formation in pressurized swirl flames by laser measurements of temperature, flame structures and soot concentrations," Proceedings of the Combustion Institute, Vol. 35, No. 3, 2015, pp. 3373-3380. https://doi.org/10.1016/j.proci.2014.05.135.

[28] Koo, H., Raman, V., Mueller, M. E., and Geigle, K.-P., "LES of a sooting flame in a pressurized swirl combustor," 54th AIAA Aerospace Sciences Meeting, AIAA, San Diego, California, USA, 2016. https://doi.org/10.2514/6.2016-2123

[29] Koo, H., Hassanaly, M., Raman, V., Mueller, M. E., and Peter Geigle, K., "Large-Eddy Simulation of Soot Formation in a Model Gas Turbine Combustor," Journal of Engineering for Gas Turbines and Power, Vol. 139, No. 3, 2017 , p. 031503. https://doi.org/10.1115/1.4034448.

[30] Franzelli, B., Riber, E., Cuenot, B., and Ihme, M., "Numerical Modeling of Soot Production in Aero-Engine Combustors Using Large Eddy Simulations," Proceedings of the ASME Turbo Expo 2015: Turbine Technical Conference and Exposition. Volume 4B: Combustion, Fuels and Emissions, ASME, Montreal, Quebec, Canada, 2015, p. V04BT04A049. https://doi.org/10.1115/GT201543630

[31] Franzelli, B., Vié, A., and Darabiha, N., "A three-equation model for the prediction of soot emissions in LES of gas turbines," Proceedings of the Combustion Institute, Vol. 37, No. 4, 2019, pp. 5411-5419. https://doi.org/10.1016/j.proci.2018.05.061

[32] Eberle, C., Gerlinger, P., Geigle, K. P., and Aigner, M., "Numerical Investigation of Transient Soot Evolution Processes in an Aero-Engine Model Combustor," Combustion Science and Technology, Vol. 187, No. 12, 2015, pp. $1841-1866$. https://doi.org/10.1080/00102202.2015.1065254

[33] Eberle, C., Gerlinger, P., Geigle, K. P., and Aigner, M., "Toward finite-rate chemistry large-eddy simulations of sooting swirl flames," Combustion Science and Technology, Vol. 190, No. 7, 2018, pp. 1194-1217. https://doi.org/10.1080/00102202.2018. 1443444

[34] Rigopoulos, S., "Modelling of Soot Aerosol Dynamics in Turbulent Flow," Flow, Turbulence and Combustion, Vol. 103, No. 3, 2019, pp. 565-604. https://doi.org/10.1007/s10494-019-00054-8 